\title{
Volitional hamstring force production during closed kinetic chain lower extremity exercise in supine position: a randomized controlled trial
}

\begin{abstract}
Purpose and hypothesis: The purpose of this study is to quantify and compare force production of the biceps femoris and semi-membranous muscles during the performance of supine, closed chain lower extremity strengthening exercises. Volitional force production and joint position were investigated during specified closed chain lower extremity activities including: supine hemi bridge, elevated hemi bridge and heel slide Hemi Bridge.
\end{abstract}

Materials and methods: This study consisted of sixty participants recruited from various cohorts of the college student population via convenience sampling method. Subjects were randomized by lottery selection and evenly distributed to one of three exercise groups: supine hemi bridge $(\mathrm{n}=20)$, elevated hemi bridge $(\mathrm{n}=20)$ and heel slide hemi bridge $(\mathrm{n}=20)$. All exercises were performed three times upon the subject's dominant lower extremity. In each group, force production of the biceps femoris and semi-membranous muscles were measured using a wireless electromyogram (EMG) (2016 DELSYS Trigno ${ }^{\mathrm{TM}}$ Sensor System) unit with surface electrodes. Participant exercise performance was also monitored during each exercise by use of a digital postural assessment tool (2016 Postureco ${ }^{\mathrm{TM}}$ Posture Screen Mobile).

Results: Total 50 participants ( 23 males and 27 females) completed the study. Semimembranous force productions were $3.39,3.85$ and $3.72 \mathrm{mV}$ on the supine, elevated and heel slide hemi bridge exercises respectively. Biceps femoris force productions were $5.79,4.50$ and $4.75 \mathrm{mV}$ on the supine, elevated and heel slide hemi bridge exercises respectively The results of the one-way ANOVA for semi-membranous and biceps femoris force production were $\mathrm{p}=0.890$ and $\mathrm{p}=0.266$ respectively, indicating no significant differences between the three exercises.

Conclusion: The supine hemi bridge, elevated hemi bridge and heel slide hemibridge were equally effective in activating the semi-membranous and biceps femoris during supine, closed chain lower extremity strengthening exercises. However, variances among EMG data indicate distinct clinical implications on exercise prescription.

Keywords: hamstring, force production, closed chain exercise, rehabilitation, hemibridge
Volume 2 Issue 4 - 2017

\author{
Kellie Carpenter, Stefan Paul, Ilona Komet, \\ Ayse Ozcan Edeer \\ Doctor of Physical Therapy Program, Dominican College, USA
}

Correspondence: Ayse Ozcan Edeer, Assistant Professor, Dominican College, USA, Tel 8458487615, Fax 8453984892, Email ayse.edeer@dc.edu

Received: October 29, 2017 | Published: November 24, 2017

\section{Introduction}

The hamstring muscle group is a vital constituent to injury prevention and lower extremity rehabilitation. During closed chain activity, hamstring muscle fibers influence dynamic interaction between the pelvis, sacrum, femur and lower leg. The hamstring muscle group functions as a frontal and transverse plane stabilizer, influencing knee flexion deceleration and eccentric extension during forward propulsion. ${ }^{1-3}$ The hamstring muscle group is most often injured during high-speed propulsion activities most notably among track runners, football players and hockey players. Consequently, the biceps femoris in particular is considerably vulnerable to strain or injury during moments of acceleration. ${ }^{3-5}$ Heiderscheit et al. ${ }^{6}$ further noted that the incidence of hamstring re-injury can be attributed to chronic musculotendinous shortening secondary to previous injury. ${ }^{6}$ According to Sherry et al., ${ }^{7}$ hamstring injuries often require significant recovery time and exhibit a long period of increased susceptibility. ${ }^{7}$
Closed chain eccentric loading of the biceps femoris has been shown to reduce the incidence of hamstring injury. ${ }^{1,7}$ Unilateral eccentric exercises that incorporate volitional control of both hip and knee motions have demonstrated preventive value as well. The researchers recommended that rehabilitation programs focus on high load eccentric contractions at the knee joint while maintaining the hip joint in a flexed position, in order to increase the elongation stress of the hamstring muscles. ${ }^{4,8}$ Heiderscheit et al. ${ }^{6}$ stated that eccentric strength training following a hamstring injury could effectively restore an optimum musculotendon length-tension ratio, thereby reducing the risk of re-injury. ${ }^{6}$ Studies have provided conclusive evidence regarding hamstring involvement throughout preventative and restorative lower extremity exercise protocols..$^{4,6-8}$ However, quantified differences in hamstring activation during weight bearing activities have not been thoroughly examined within associated literature. 
This study examines the force production of the biceps femoris and semi-membranous during performance of prescribed exercises (supine hemi bridge, elevated hemi bridge and heel slide hemi bridge) using EMG biofeedback in young, healthy subjects. The purpose of this study is to establish criteria for evidence based exercise prescription, intended to maximize hamstring activation during lower extremity strengthening protocols.

The results of this study may be applied to existing preventative, restorative and function-based treatment protocols. In addition to comparatively quantifying maximal hamstring activation during closed chain supine exercises, this study will qualitatively appraise the impact of positional changes on hamstring recruitment. Clinicians and other health care professionals may utilize the results of this study to implement evidence-based recommendations on lower extremity exercises designed to maximally (or sub-maximally) recruit biceps femoris and semi-membranous muscle fibers.

\section{Materials and methods}

This study consisted of sixty participants recruited from various cohorts of the College students by using convenience sampling. An approval was obtained prior to data collection from the Institutional Review Board (IRB\#2016-0322-02). Participants were selected based upon the following inclusion criteria: ages $18-40$ with no history of lower extremity injury or surgery within the past six months. Participants were excluded from the study based upon subjective reports of positional vertigo and degenerative bone/joint disease. Informed consent and demographic information (age, sex, height and dominant lower extremity) were obtained from each participant (Table 1). Subjects were randomized by lottery selection and evenly distributed to one of three exercise groups: supine hemi bridge $(n=20)$, elevated hemi bridge $(n=20)$ and heel slide hemi bridge $(n=20)$

Table I Participants demographics

\begin{tabular}{lll}
\hline & Mean \pm SD & $\mathbf{n}$ \\
\hline Age & $24.7 \pm 4.61$ & - \\
Gender & - & 23 Males/27 females \\
Height & $66.32 \pm 3.71$ & - \\
Dominant leg & - & 59 right/1 left \\
\hline
\end{tabular}

Materials for assessment included an electromyography (EMG) biofeedback unit with wireless surface electrodes (2016 DELSYS Trigno ${ }^{\mathrm{TM}}$ Sensor System), ${ }^{9}$ a standard examination table and a digital postural assessment tool (2016 Postureco ${ }^{\mathrm{TM}}$ Posture Screen Mobile). ${ }^{10}$ Investigators examined semi-membranous and biceps femoris muscle activity using EMG biofeedback during hemi bridge exercises. Investigator 1 was assigned to strategic placement of wireless EMG electrodes onto the biceps femoris and semi-membranous muscles of each subject's dominant lower extremity. One electrode was situated along the muscle belly of the biceps femoris, located midway between the ischial tuberosity and lateral epicondyle of tibia. A second electrode was situated along the muscle belly of the semi-membranous, located at the midpopliteal crest. Based on the origin/insertion of the biceps femoris, the participants were cued to maintain a posterior pelvic tilt throughout the range of motion to ensure mechanical advantage and to minimize risk of injury during the study. ${ }^{11,12}$
EMG values were recorded at rest and during the performance of a single muscle activity specified by each participant's treatment group. Data collection was done afternoon for all participants in order to avoid timing as a confounding factor. Investigator 2 was relegated to the provision of verbal and demonstrative instruction for each participant, prior to each exercise. All activities were performed on a flat and even surface. All participants were able to maintain a supine position throughout the data collection process, without complication. Volitional contractions were repeated for a total of three repetitions with a rest period of thirty seconds between each movement. Force production data was recorded during each volitional contraction. The EMG values attained during the second repetition were then used for statistical analysis.

Investigator 3 appraised each subject using a digital postural assessment tool, with particular attention to pelvic displacement and postural alignment during activity performance (Figures 1-3). Statistical Package for the Social Sciences (SPSS) Version 24 was used for statistical analysis. The one-way analysis of variance (ANOVA) was used to determine whether there are any statistically significant differences between the means of three different bridges performed throughout the study. Wilcoxon Signed Rank test was used to compare force production of semi-membranous and biceps femoris. The level of statistical significance was set at $p<0.05$.

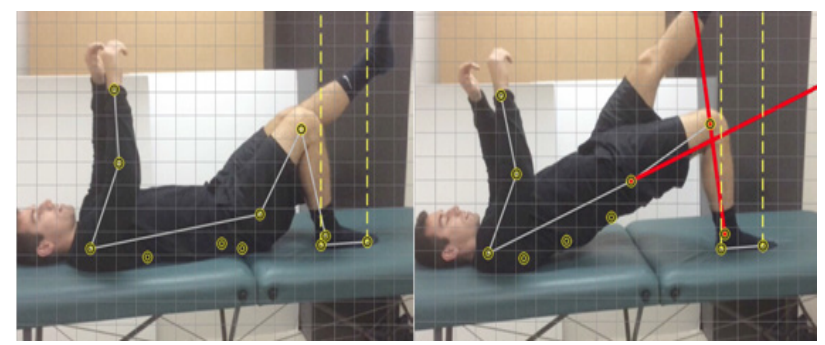

Figure I Digital postural assessment during supine hemibridge exercise.

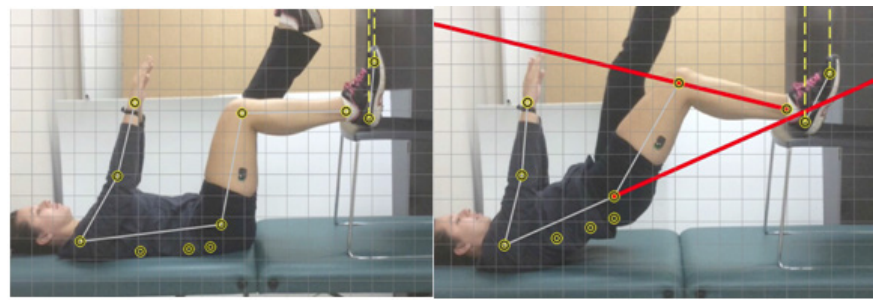

Figure 2 Digital postural assessment during elevated hemibridge exercise.

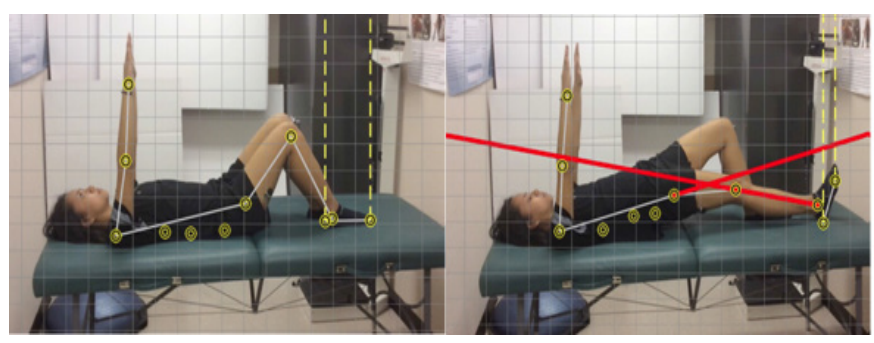

Figure 3 Digital postural assessment during heel slide hemibridge exercise. 


\section{Results}

EMG data were collected from 60 participants during closed chain volitional contractions of the biceps femoris and the semimembranous muscles. Based upon exclusion criteria 6 subjects of the 60 participants were excluded from the data analysis; elevated hemi bridge $n=1$, supine hemi bridge $n=2$, heel slide hemi bridge $n=3$ (injury on lower extremities in last 6 months $=2$, positional vertigo $=2$, degenerative bone disease $=2$ ). After analyzing the raw EMG data using SPSS in a one-way ANOVA, 4 more participants were excluded as outliers; supine hemi bridge $n=2$ and heel slide hemi bridge $n=2$. Data from total 50 participants were used for statistical analysis; supine hemi bridge $=16$, elevated hemi bridge $=19$, heel slide hemi bridge $=15$ (Figure 4 ).

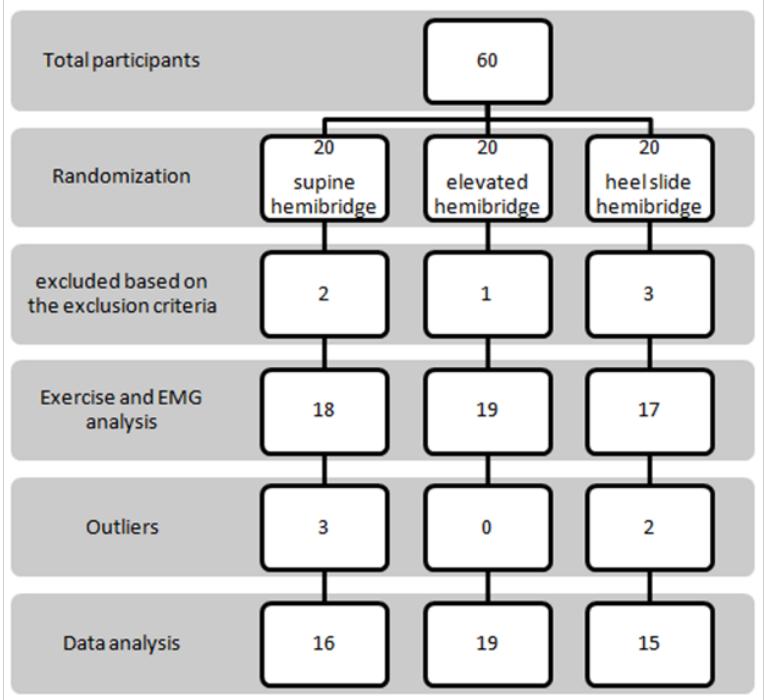

Figure 4 Consort flow.

Semi-membranous force production was $3.39,3.85$ and $3.72 \mathrm{mV}$ on the supine, elevated and heel slide hemi bridge exercises respectively. The results of the one-way ANOVA for semi-membranous force production was $\mathrm{p}=0.890$, indicating no significant differences between the three exercises. Biceps femoris force production was 5.79, 4.50 and $4.75 \mathrm{mV}$ on the supine, elevated and heel slide hemi bridge exercises respectively. The results of the one-way ANOVA for biceps femoris force production was $\mathrm{p}=0.266$, indicating no significant difference between the three exercises. However, variances among quantitative values indicated potential clinical significance (Table 2). When we compare the semi-membranous and biceps femoris force production on these three exercises, we observed that biceps femoris force production was higher than semi-membranous force production on three exercises. However these differences were not statistically significant $(\mathrm{p}>0.05)$ (Table 3).

Further appraisal of each average EMG values revealed distinct variances in force production. Average semi-membranous EMG readings from highest to lowest were: elevated hemi bridge, heel slide Hemi Bridge and supine hemibridge. Average biceps femoris EMG readings from highest to lowest were: supine hemi bridge, heel slide hemibridge and elevated hemi bridge. Combined force productions of the semi-membranous and biceps femoris were maximized during the supine hemi bridge, followed by the heel slide hemibridge and the elevated hemi bridge, respectively (Graphic 1).

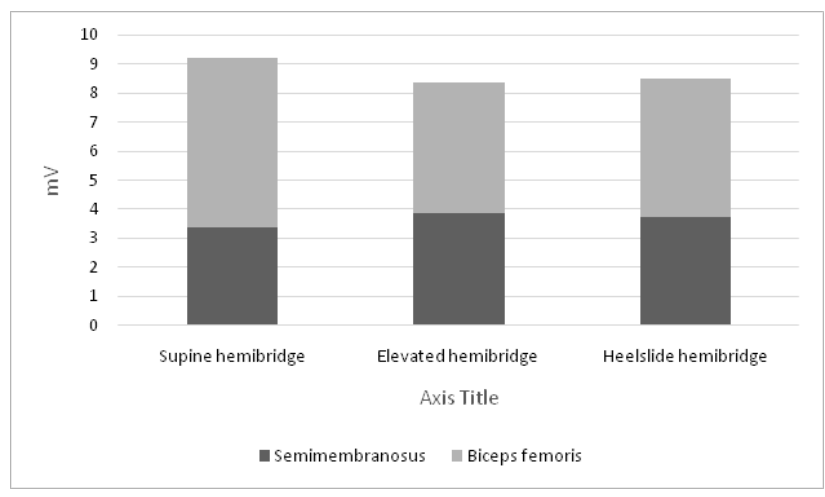

Graphic I Combined force production of semi-membranous and biceps femoris EMG muscle activity during supine, elevated and heel slide hemibridge exercises (root mean squared average).

Table 2 The comparison force production on the supine, elevated and heel slide hemibridge exercises (One-way ANOVA) (root mean squared average)

\begin{tabular}{lllll}
\hline & $\begin{array}{l}\text { Supine } \\
\text { hemibridge }\end{array}$ & $\begin{array}{l}\text { Elevated } \\
\text { hemibridge }\end{array}$ & $\begin{array}{l}\text { Heel slide } \\
\text { hemibridge }\end{array}$ & $\mathbf{p}$ \\
\hline $\begin{array}{l}\text { Semi- } \\
\text { membranous } \\
(\mathrm{mV})\end{array}$ & $3.39 \pm 2.73$ & $3.85 \pm 3.10$ & $3.72 \pm 2.47$ & 0.89 \\
$\begin{array}{l}\text { Biceps } \\
\text { femoris }(\mathrm{mV})\end{array}$ & $5.79 \pm 2.31$ & $4.50 \pm 2.51$ & $4.75 \pm 2.15$ & 0.266 \\
\hline
\end{tabular}

Table 3 Comparison of force production of semi-membranous and biceps femoris (Wilcoxon Signed Rank) (root mean squared average)

\begin{tabular}{llll}
\hline & $\begin{array}{l}\text { Semi- } \\
\text { membranous }\end{array}$ & Biceps femoris & P \\
\hline Supine hemibridge $(\mathrm{mV})$ & $3.39 \pm 2.73$ & $5.79 \pm 2.31$ & 0.051 \\
Elevated hemibridge $(\mathrm{mV})$ & $3.85 \pm 3.10$ & $4.50 \pm 2.51$ & 0.167 \\
Heel slide hemibridge $(\mathrm{mV})$ & $3.72 \pm 2.47$ & $4.75 \pm 2.15$ & 0.363 \\
\hline
\end{tabular}

\section{Discussion}

This study quantified and compared maximal force production of the biceps femoris and semi-membranous during three supine, closed chain exercises. EMG data was collected throughout the concentric and eccentric phases. According to statistical analysis, the supine hemi bridge, heel slide hemibridge and elevated hemi bridge were equally effective in activating biceps femoris and semi-membranous. Although statistical differences in hamstring activation were marginal and insignificant, variances among EMG readings indicate distinct clinical implications on exercise prescription. Appropriate application of the supine hemi bridge, elevated hemi bridge, or heel slide hemibridge may differ depending on the desired functional outcome of each closed chain exercise. EMG data suggests clinically meaningful differences between exercises with respect to volitional recruitment of biceps femoris and semi-membranous. Combined volitional force productions of the semi-membranous and biceps femoris were maximized during the supine hemi bridge, followed by the heel slide hemibridge and the elevated hemi bridge, respectively. The biceps femoris produced maximal (isolated) force production during the supine hemi bridge, followed by the heel slide hemibridge and the 
elevated hemi bridge. The semi-membranous produced maximal (isolated) force production during the elevated hemi bridge, followed by the heel slide hemibridge and the supine hemibridge.

Based upon established evidence of hamstring influences on gait and propulsion, the heel slide hemibridge exhibits qualities most conducive of functional carryover. Through observation and performance appraisal, the heel slide hemibridge was found to adequately challenge lower extremity sequencing, reciprocal motion, bilateral activity and static/dynamic stability. Maximal biceps femoris force production was also achieved during the heel slide hemibridge, as evidenced by EMG data.

In addition to lower extremity arthrokinematics, the hamstring muscle group influences pelvic position in tandem with ipsilateral gluteal and oblique abdominal fibers. This muscular complex is the driving force behind static and dynamic postural stability. The hamstring muscle group, gluteal complex and oblique abdominal fibers exert tri-planar influences on spinal curvature, innominate rotation and acetabular femoral alignment. Coordination of the lumbopelvic region during lower extremity activity allows the hamstring muscles to function at safe lengths and loads throughout reciprocal and alternating movements. Therefore, neuromuscular control of the lumbopelvic region is required to enable optimal function of the hamstrings during gait and propulsion activities. ${ }^{3,6}$ Mechanical function of the biceps femoris also indicates that a posterior pelvic tilt should be maintained during each bridging activity to promote safety and mechanical advantage of the hamstring musculature.

Due to influences on pelvic, hip and lower leg alignment, findings may also be applied to hamstring activation in clinical management of populations including: musculotendinous/ligamentous knee repair, sacroiliac joint dysfunction and low back pain. Future studies may examine resisted or positional progression into squat, dead lift or sprinting sequences. In addition to exercise progression, adaptation of the digital postural assessment tool appropriate to horizontal and supine positions may improve clinical use and applicability.

The limitations of this study include relatively small sample size, difficulty engaging participants, lack of info regarding the exercise habits of the participants and lack of comparative statistics for baseline hamstring force production. Wireless EMG leads were positioned according to established research conducive of maximal and accurate measurement of muscle activity. Consistency of subject posture during exercise performance was maintained throughout the data collection process through verbal and tactile cues conducive of a sustained posterior pelvic tilt. The absence of a preset supine-lateral movement screen limited the quantitative measurements of joint position during activity performance. Participant randomization and musculoskeletal-based exclusion criteria further ensured the validity of the research design. Instrumentation of measurement devices was exclusive to each individual researcher in order to limit potential confounding variables during the data collection process.

\section{Conclusion}

The supine hemi bridge, heel slide hemibridge and elevated hemi bridge were equally effective in activating the semi-membranous and biceps femoris during supine, closed chain lower extremity strengthening exercises. The exercises examined in this study may be referenced during prevention or rehabilitation protocols of hamstring strain and associated sequela. Although statistical differences in hamstring activation were marginal and insignificant, variances among EMG readings indicate distinct clinical implications on exercise prescription. Appropriate application of the supine hemi bridge, heel slide hemibridge and elevated hemi bridge may differ depending on the desired functional outcome.

\section{Acknowledgements}

None.

\section{Conflict of interest}

The author declares no conflict of interest.

\section{References}

1. Goldman EF, Jones DE. Interventions for preventing hamstring injuries Cochrane Database Syst Rev. 2010;20(1):CD006782.

2. Gracovetsky S. A hypothesis for the role of the spine in human locomotion: a challenge to current thinking. J Biomed Eng. 1985;7(3):205-216.

3. Shadmehr A, Jafarian Z, Talebian S. Changes in recruitment of pelvic stabilizer muscles in people with and without sacroiliac joint pain during the active straight-leg-raise test. J Back Musculoskelet Rehabil. 2012;25(1):27-32.

4. Monajati A, Zabala LE, Sampson GM, et al. The effectiveness of injury prevention programs to modify risk factors for non-contact anterior cruciate ligament and hamstring injuries in uninjured team sports athletes: a systematic review. PLoS One. 2016;11(5):e0155272.

5. Opar MDA, Williams MD, Shield AJ. Hamstring strain injuries. Sports Medicine. 2012;42(3):209-226.

6. Heiderscheit BC, Sherry MA, Silder A, et al. Hamstring strain injuries: recommendations for diagnosis, rehabilitation, and injury prevention. $J$ Orthop Sports Phys Ther. 2010;40(2):67-81.

7. Sherry MA, Best TM, Silder A, et al. Hamstring strains: basic science and clinical research applications for preventing the recurrent injury. Strength \& Conditioning Journal. 2011;33(3):56-71.

8. Guex K, Millet GP. Conceptual framework for strengthening exercises to prevent hamstring strains. Sports Med. 2013;43(12):1207-1215.

9. http://delsys.com/Attachments_pdf/manual/MAN-012-2-7.pdf

10. http://postureanalysis.com/mobile/

11. Kotila K, Sveinsson T, Árnason Á. Evidence-based testing of the hamstring muscles using wireless surface EMG. British Journal of Sports Medicine. 2011;45(4):358.

12. Wingerden VJP, Vleeming A, Snijders CJ, et al. A functional anatomical approach to the spine-pelvis mechanism: Interaction between the biceps femoris muscle and the sacrotuberous ligament. Eur Spine J. 1993;2:140144. 